Retraction

\title{
Mast cells induce epithelial-to-mesenchymal transition and migration in non-small cell lung cancer through IL-8/Wnt/ $\beta$-catenin pathway
}

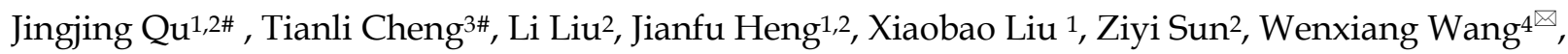
Kunyan $\mathrm{Li}^{1 凶}$, Nong Yang ${ }^{2 凶}$

1. Department of Clinical Pharmaceutical Research Institution, Hunan Cancer Hospital, Affiliated Tumor Hospital of Xiangya Medical School of Central South University, Changsha, 410008, China

2. Department of Lung Cancer and Gastroenterology, Hunan Cancer Hospital, Affiliated Tumor Hospital of Xiangya Medical School of Central South University, Changsha, 410008, China

3. Thoracic Medicine Department 1, Hunan Cancer Hospital, Affiliated Tumor Hospital of Xiangya Medical School of Central South University, Changsha, 410008, China

4. The Second Department of Thoracic Surgery, Hunan Cancer Hospital, Affiliated Tumor Hospital of Xiangya Medical School of Central South University, Changsha, 410008, China

\# Jingjing Qu and Tianli Cheng contributed equally to this work.

$\triangle$ Corresponding authors: Prof. Wenxiang Wang, Department of the Second Department of Thoracic Surgery, Hunan Cancer Hospital, Affiliated Tumor Hospital of Xiangya Medical School of Central South University, Changsha, 410008, China. Email: 2460585948@qq.com. Prof. Kunyan li, Department of Clinical Pharmaceutical Research Institution, Hunan Cancer Hospital, Affiliated Tumor Hospital of Xiangya Medical School of Central South University, Changsha, 410008, P.R. China. Email: Lkunyan@163.com. Prof. Nong Yang, Department of Lung Cancer and Gastroenterology, Hunan Cancer Hospital, Affiliated Tumor Hospital of Xiangya Medical School of Central South University, Changsha, 410008, P.R. China. Email: yangnong0217@163.com.

(C) Ivyspring International Publisher. This is an open access article distributed under the terms of the Creative Commons Attribution (CC BY-NC) license (https://creativecommons.org/licenses/by-nc/4.0/). See http://ivyspring.com/terms for full terms and conditions.

Published: 2019.09 .02

Corrected article: J Cancer 2019; 10(16):3830-3841. doi:10.7150/jca.29953.

After careful consideration, we (the authors) recognized that our paper contains many defects both in English and content (such as use of the same picture and unclear description in the Materials and Methods).

Our co-first author was, unfortunately, an inexperienced author. Our English and writing skills were inadequate. There are some unintentional errors in the paper, and we had not addressed them fully. We assure you that the results and the data are original, based on clinical findings, including the in vivo mouse model and in vitro cell experiment.

We would like to withdraw this paper in order to be responsible for the readers of the journal. All of the authors have agreed to withdraw the paper. 